\title{
The origin of spacetime topology and generalizations of quantum field theory
}

\author{
Ulvi Yurtsever \\ Department of Physics \\ University of California \\ Santa Barbara, CA 93106
}

October, 1992

UCSBTH-92-45

\begin{abstract}
The research effort reported in this paper is directed, in a broad sense, towards understanding the small-scale structure of spacetime. The fundamental question that guides our discussion is "what is the physical content of spacetime topology?" In classical physics, this question has a natural and simple answer: spacetime (as a topological space) is a bookkeeping device that we invent to make the description of classical fields (the observables) easier. More precisely, if spacetime, $(X, \tau)$, has sufficiently regular topology, and if sufficiently many fields exist to allow us to observe all continuous functions on $X$, then this collection of continuous functions uniquely determines both the set of points $X$ and the topology $\tau$ on it. Naturally, however, this answer does not
\end{abstract}


yield any clues into why our spacetime is observed to have a very special (i.e. smooth manifold) topology down to the smallest scales we can probe, or into whether this smooth manifold structure persists indefinitely, at length scales smaller than the smallest observed so far. To explore these queries, we are led to consider the original question in the context of quantum, rather than classical field theory. After all, in the real world physical fields (the observables) are not classical (continuous functions) but quantum operators, and the fundamental observable is not the collection of all continuous functions but the local algebra of quantum field operators. Presently the only examples of local quantum field algebras that we know how to construct rigorously (apart from some two-dimensional models) are the operator algebras of free (linear) quantum fields propagating on a smooth, globally hyperbolic spacetime. Since this class of examples is too small, we find it necessary to generalize the algebraic notion of "quantum field" in such a way that it becomes possible to talk about quantum field theory on an arbitrary (not necessarily smooth) topological space on which no notion of spacetime metric exists a priori. [One interesting offshoot of this generalization is an algebraic framework for linear quantum field theory on non-globally-hyperbolic spacetimes (e.g. spacetimes with naked singularities or closed timelike curves), which is the subject of a separate paper submitted elsewhere.] In pursuing the original problem further, we develop a still wider generalization of quantum field theory; this ultimate generalization dispenses with the fixed background topological space altogether and proposes that the fundamental observable should be taken as a lattice (or more specifically a "frame," in the sense of set theory) of closed subalgebras of an abstract $C^{*}$ algebra. Our discussion concludes with the definition and some elementary properties of these "quantum lattices" and "quantum frames." 


\section{Introduction}

Spacetime, general relativity teaches us, is not a fixed, pre-determined background on which physical processes take place, but a dynamical entity itself. In classical (relativistic) physics, the dynamical content of spacetime is primarily geometric, with the metric and matter fields tied together as described by Einstein field equations. In quantum physics, it is widely believed that not only the geometry of spacetime but also its topology must be subject to dynamical fluctuations, governed by an as yet unknown quantum theory of gravity. The scale at which these fluctuations become significant is set by the Planck length $\left(l_{P} \sim 10^{-33} \mathrm{~cm}\right)$. The small scale structure of spacetime at or below the Planck length is bound to be very different from that of a locally Euclidean, four-dimensional Lorentzian manifold, the structure currently observed to be accurate down to at least about $10^{-16} \mathrm{~cm}$.

The imminent breakdown of the macroscopic structure of spacetime at sufficiently small length scales (too small to be accessible by experiments in the foreseeble future) forces us to take one of two positions: Either the basic manifold structure (but not necessarily the four-dimensional Lorentzian geometry) of spacetime is fundamental and accurate down to arbitrarily small scales, or, the structure of spacetime below some small length scale $\left(\geq l_{P}\right)$ is fundamentally different from that of a point set with manifold topology. If the first position is adopted, there is in principle no reason to expect that our basic notions about physical space will have to be revised radically in order to understand the quantum theory of spacetime. The standard, differentialgeometry-based approach to classical field theory, along with the universally accepted principles of quantum mechanics, have to be capable, eventually, of achieving this ultimate quantization. This attitude is implicit in much of the current work on quantum gravity, in both string theory and the canonical quantization approach.

By contrast, if the second position is adopted, then one cannot escape the conclusion that understanding the full quantized structure of spacetime will have to involve extensive and radical changes in our basic notions about space, field theory and gravity. The argument for this conclusion can be stated briefly as follows: In every known example of quantum field theory on a fixed (manifold) background, the equations of motion for the classical fields continue to hold after quantization. Although the expectation values of the fields do not, in general, satisfy the classical field equations, the field op- 
erators themselves (constructed as operator-valued generalized functions on the background) always do. (We are assuming, of course, that the nonlinear interaction terms involving the fields are suitably regularized to make proper sense of the products of field operators. The difficulties associated with this step are notorious; however, these difficulties are technical, not fundamental.) In other words, in standard quantum field theory the equations of motion that form the starting point for quantization are fundamentally valid, not only classically but also in the quantum theory. If, on the other hand, our goal is to quantize spacetime itself (or, what amounts to the same thing, to quantize gravity), and if spacetime does not have manifold structure at small-enough length scales, then the classical field equations, defined as they are in terms of the macroscopic, manifold "limit" of spacetime, have absolutely no chance of being fundamental: at the quantum level not even the formulation of these equations makes sense. The straightforward quantization of any classical field theory (whether that theory is general relativity, string theory, or some other extension of general relativity) cannot bring us a final understanding of the quantum structure of spacetime. (For a poignant comparison, consider the understanding one would gain about the small-scale structure of a fluid from the quantization of the Navier-Stokes equations.) In short, if we take the position that the fundamental, small-scale structure of spacetime is not that of a manifold, then no formalism based on differential geometry can be the correct quantum theory of gravity.

In a series of papers, of which this is the first one, we will adopt this second position and explore some of its consequences from a specific, and hopefully novel, point of view. There are other, previous studies of spacetime structure which adopted a similar attitude towards the ultimate nature of quantum gravity. Perhaps the best-known among these are the spin-networks idea of Penrose ([1]), the work on causal sets by Sorkin and others ([2]), and the more recent work of Isham ([3]) on quantum topology. Our approach differs fundamentally from these earlier studies in one respect: We will not develop, at the outset, a specific, "discrete" structure, postulate it to be the fundamental small-scale structure of spacetime, and proceed to discuss its consequences. Instead we ask: what is the essential content of our notion of "space" in physics; or, in more precise terms, what is the physical origin of spacetime topology? In essence our answer is simple: Spacetime is not observable directly; only fields are. Spacetime, then, is a "bookkeeping" device, one of indispensible convenience for describing the dynamics of the physical fields we observe, but not as fundamental as the fields themselves. This is the guiding principle of our approach, and it will frame our discussion throughout this and the later papers in the series. In this first paper, we will 
mainly develop the foundations for our viewpoint, i.e. explain how spacetime structure can be regarded naturally as "derived" from field theory. We start with the case of classical fields.

\section{The origin of spacetime topology in classical physics}

The most general proper context in which the notion of spacetime structure can be placed in classical field theory is the category of topological spaces and continuous maps. By this we mean that spacetime is a set, $X$, endowed with a topology, $\tau$, and fields are continuous functions from $(X, \tau)$ into other, target topological spaces. By contrast, in quantum theory the most general category for spacetime structure is in fact bigger than the topological category as we will see shortly.

To render our discussion here more transparent we will make two simplifying assumptions: First we assume that there exist sufficiently many physical fields on spacetime to allow us to observe the family, $\mathcal{F}$, of all continuous real-valued functions on $(X, \tau)$. [Clearly, "observable" functions include not only the fields themselves, but also a large class of (e.g. piecewise analytic) functions of the (components of) fields.] Next, we will assume that it is possible to distinguish by observation whether a given collection of real numbers, $\left\{c_{f} \mid f \in \mathcal{F}\right\}$, corresponds to the values assumed by the observables in $\mathcal{F}$ at a point of $X$; i.e., we assume that given $\left\{c_{f}\right\}$ we can decide whether there exists a point $x \in X$ such that $c_{f}=f(x) \forall f \in \mathcal{F}$. More sophisticated approaches that do away with these assumptions are possible; however, we do not expect that our main conclusion will be altered by relaxing the above assumptions.

The collection $\mathcal{F}$ is the fundamental observable associated with the spacetime $(X, \tau)$. How can we reconstruct $(X, \tau)$ from the knowledge of $\mathcal{F}$ ? Obviously, we need to recover both the point-set $X$ and the topology $\tau$ on it. This can be done if and only if the following two conditions are satisfied:

(i): The collection $\mathcal{F}$ separates points; that is, for any $x, y \in X, x \neq y$, there exists a continuous function $f$ in $\mathcal{F}$ such that $f(x) \neq f(y)$.

(ii): The topology $\tau$ is the weakest topology on $X$ that makes every $f \in \mathcal{F}$ continuous; in other words, $\tau$ is generated by subsets of $X$ of the form $f^{-1}(J)$, where $f \in \mathcal{F}$, and $J \subset \mathbf{R}$ is open in $\mathbf{R}$.

In other words, if $(X, \tau)$ satisfies conditions (i) and (ii), then the knowledge 
of the set of all classical observables, $\mathcal{F}$, is equivalent to the knowledge of both $X$ and $\tau$. In fact, this is precisely the way we "deduce" the topology of physical space in classical physics: all neighborhood relationships are determined relative to the measurements of fields, and fields are declared to be continuous functions at the outset.

Which topological spaces satisfy the conditions (i) -(ii)? To answer this question, it is convenient to recast the conditions (i) and (ii) in a slightly more elegant form as follows: By composing each continuous function in $\mathcal{F}$ with a fixed homeomorphism of $\mathbf{R}$ onto the unit interval $(0,1)$, we can, without loss of generality, replace $\mathcal{F}$ with the collection of all (bounded) continuous functions $X \longrightarrow[0,1]$ (which collection we will still denote by $\mathcal{F}$ ). Let $I$ denote the closed interval $[0,1]$, and let $I_{\mathcal{F}} \equiv \prod_{f \in \mathcal{F}} I$, the Cartesian product of $\mathcal{F}$-many copies of $I$, with one copy for each distinct $f \in \mathcal{F} . I_{\mathcal{F}}$ is a compact Hausdorff space under the natural product topology. We can now construct the following canonical map

$$
\begin{aligned}
i & : \quad X \longrightarrow I_{\mathcal{F}}, \\
i & : \quad x \mapsto i(x) \in I_{\mathcal{F}} \text { where }[i(x)]_{f}=f(x) \quad \forall f \in \mathcal{F} .
\end{aligned}
$$

It is easy to see that $i$ is continuous in general, and that the conditions (i)-(ii) are equivalent to the statement that $i$ is an imbedding, i.e. a homeomorphism onto its image $i(X) \subset I_{\mathcal{F}}$. Therefore, in Eq. (1) we have an explicit picture of the reconstruction of $(X, \tau)$ from the observed data $\mathcal{F}$ (and the subset $i(X) \subset I_{\mathcal{F}}$, which is distinguishable in $I_{\mathcal{F}}$ by our assumption). The question we posed at the beginning of the paragraph now becomes: for which spaces $(X, \tau)$ the map $i$ given by Eq. (1) is an imbedding? The answer is well known in topology, and these are precisely the so-called Tychonoff spaces, i.e. Hausdorff spaces $X$ with the property that for any closed subset $A$ and a point $x$ not in $A$ there exists a continuous function $f: X \longrightarrow \mathbf{R}$ such that $f(A)=a$ and $f(x)=b$, where $a \neq b$ (see [4], Sect. 14). Tychonoff spaces are regular (in fact $T_{3}$ since $T_{2}$ holds by definition), but not every $T_{3}$ space is Tychonoff. Subspaces and products of Tychonoff spaces are Tychonoff, and every locally compact Hausdorff space (hence every manifold) and all metric spaces are Tychonoff. An interesting property is that a connected Tychonoff space is either a trivial, one-point space, or contains uncountably many points.

We have thus demonstrated that in classical field theory spacetime topology can be recovered completely from a knowledge of the fields (continuous functions) provided the background, $(X, \tau)$, is a Tychonoff space. This answer is unsatisfactory for a number of reasons: First of all, it does not 
bring any insight into why our spacetime is observed, at least down to the smallest length scales we can probe, to have a smooth manifold structure. Although Tychonoff spaces and manifolds have in common the property that any nontrivial, connected subspace is uncountable, there remains a large "evolutionary" distance between a typical Tychonoff space and a manifold. Indeed, general Tychonoff spaces are not even metrizable. Our answer is also unsatisfactory in that it does not provide an interesting alternative to manifold structure that might serve as a candidate for the small-scale structure of spacetime. Tychonoff spaces are more general than manifolds, but they hardly seem promising as candidates for the fundamental structure of spacetime at arbitrarily small length scales.

These deficiencies, however, are expected, or should have been expected, because the above questions which our approach so far failed to answer are quantum-mechanical in nature, not classical. After all, the expectation that spacetime at small-enough length scales is likely to have nonmanifold structure has a quantum-mechanical motivation. Also, in the real world physical fields (the observables) are not classical, continuous functions but operator-valued quantum fields. We have to formulate our approach in the context of quantum field theory to be able to address the fundamental issues relating to small-scale spacetime structure.

We have argued that the only observable associated with topological structure in classical field theory is the collection $\mathcal{F}$ of all continuous, realvalued functions on spacetime. The main challenge that we will face for the rest of this paper is the formulation of a suitable analogoue of the observable $\mathcal{F}$ in quantum field theory. The best (and perhaps the only) approach to quantum field theory in which this formulation can be worked-out is the algebraic approach. For reviews of the essential aspects of the algebraic approach to quantum field theory in curved spacetime, we recommend the reader consult Refs. [5] and [6]; for an extremely brief overview see Sect. 1 of $[7]$.

Recall that the fundamental construction in the algebraic approach is the local algebra of field operators, $\mathcal{A}$, along with the "net" of closed subalgebras, $\{\mathcal{A}(U)\}$, where for each open subset $U$ in the (globally hyperbolic) spacetime $(M, g), \mathcal{A}(U)$ denotes the field operators localized in $U$. Now consider a specific example, e.g. the quantum theory of a linear Klein-Gordon field on a globally hyperbolic spacetime $(M, g)$. It is not difficult to see that if we fix a global Cauchy surface $\Sigma$ in $(M, g)$, and consider all Lorentz metrics $h$ on $M$ for which $\Sigma$ is a Cauchy surface for $(M, h)$, then the field algebras 
$\mathcal{A}$ corresponding to $(M, h)$ are all isomorphic to the original algebra $\mathcal{A}$ of $(M, g)$. The subalgebras $\mathcal{A}(U)$, on the other hand, are not mapped onto each other by the corresponding isomorphisms; i.e. the isomorphisms do not preserve the subalgebras. This means that the algebra $\mathcal{A}$ and the net structure of the subalgebras $\{\mathcal{A}(U)\}$ contain information about the topology of $M$, whereas exactly which subalgebras of $\mathcal{A}$ the $\mathcal{A}(U)$ correspond to contain the geometric information about the Lorentz metric on $M$. This observation suggests that the structure consisting of the pair $[\mathcal{A},\{\mathcal{A}(U)\}]$ is the right analogue of the observable $\mathcal{F}$ in quantum theory.

The difficulty now is that ordinarily we know how to construct local field algebras only if the background is a globally hyperbolic spacetime, and then only for linear (noninteracting) fields (with the exception of some twodimensional examples). We need a generalization of the algebraic approach that would make it possible to make sense of quantum field theory on arbitrary background topological spaces, not just on Lorentzian manifolds. In the following we will carry out such a generalization; our general notion of quantum field is abstracted from the usual algebraic notion by keeping only its most elementary, bare-bones essentials.

\section{A generalization of the algebraic framework for quantum field theory}

Let $X$ be a topological space. A "quantum field theory" on $X$ consists of an abstract $C^{*}$ algebra $\mathcal{A}$ (with identity element 1), and a map (which we will also denote by $\mathcal{A}$ ) that associates to each open subset $U$ in $X$ a closed subalgebra $\mathcal{A}(U) \subset \mathcal{A}$ such that the following two conditions hold:

(QF1): For every open subset $U \subset X \mathcal{A}(U)$ is a central $C^{*}$ algebra, and $\mathcal{A}(\{\})=\mathrm{C} \cdot 1, \mathcal{A}(X)=\mathcal{A}$.

(QF2): For any collection $\left\{W_{\alpha}\right\}$ of open subsets,

$$
\mathcal{A}\left(\bigcup_{\alpha} W_{\alpha}\right)=\overline{<\bigcup_{\alpha} \mathcal{A}\left(W_{\alpha}\right)>} .
$$

Here \{\} denotes the empty set, $\mathbf{C} \cdot 1$ is the $C^{*}$ algebra (isomorphic to the algebra $\mathbf{C}$ of complex numbers) generated by 1 , a central algebra $\mathcal{B}$ is one with the property that its center,

$$
Z(\mathcal{B}) \equiv\{x \in \mathcal{B} \mid x y=y x \forall y \in \mathcal{B}\},
$$

is equal to $\mathbf{C} \cdot 1[Z(\mathcal{B}) \cong \mathbf{C}],\langle S\rangle$ denotes the subalgebra generated by a

subset $S \subset \mathcal{A}$, and overbar denotes closure in $\mathcal{A}$. We will call the theory $\mathcal{A}$ 
"nondegenerate" if $\mathcal{A}(U)$ is strictly bigger than $\mathrm{C}$ for every nonempty open subset $U \subset X$. Property QF2 implies (but is stronger than) the well-known "net-structure" on the subalgebras $\{\mathcal{A}(U)\}$ : whenever $U \subset V$, it holds that $\mathcal{A}(U) \subset \mathcal{A}(V)$. Note that, in standard quantum field theory, property QF2 does not in general hold for the local algebra of all "observables," but it does hold when $\mathcal{A}$ consists only of (exponentiated) smeared field operators.

Now we can incorporate the notion of "locality" into our general formulation of field theory. For this, let for each point $p \in X C(p)$ denote the set

$$
\begin{aligned}
C(p) \equiv & \{q \in X \mid \nexists \text { open sets } U, V \text { such that } \\
& p \in U, q \in V \text {, and }[\mathcal{A}(U), \mathcal{A}(V)]=0\},
\end{aligned}
$$

where for $A, B \subset \mathcal{A},[A, B]$ denotes the commutator subalgebra generated by elements of the form $\{a b-b a \mid a \in A, b \in B\}$. The set $C(p)$ consists of those points $q \in X$ that can "causally communicate" with $p$ through fields in $\mathcal{A}$. [We restrict ourselves throughout to bosonic fields; hence our use of the commutator $[$,$] . It is straightforward to formulate a fermionic version of our$ discussion by replacing commutators with anti-commutators. But note that, in the fermionic case, the generators of the field algebra correspond to the smeared field operators themselves (which are already bounded) in contrast to the bosonic case, where they correspond to the exponentiated smeared fields ([5], [6]).] Some immediately obvious properties of $C(p)$ are: $q \in$ $C(p)$ iff $p \in C(q), C(p)$ is a closed subset of $X$, and, when $\mathcal{A}$ is nondegenerate, $p \in C(p) \quad \forall p \in X$ (this last result follows from QF1). A continuous curve $\gamma: \mathbf{R} \longrightarrow X$ is called a "connector" if for every $t_{0} \in \mathbf{R}$ there exists an $\epsilon>0$ such that $\gamma(t) \in C[\gamma(s)]$ for all $t, s$ in the interval $\left(t_{0}-\epsilon, t_{0}+\epsilon\right)$. (Thus defined a connector is analogous to a causal curve in spacetime.) The notion of locality for a quantum field theory $\mathcal{A}$ on $X$ is now defined in terms of the topological properties of the sets $C(p)$. Thus, we will say that $\mathcal{A}$ is "weakly local" if the following two conditions are satisfied:

(L): There exists an open neighborhood $V$ around every point $p \in X$ such that for every open neighborhood $U$ of $p$ contained in $V$ the set $U \cap[C(p) \backslash\{p\}]$ is disconnected (here \denotes set difference).

(WL): For all $p \in X C(p)$ is connected.

The theory $\mathcal{A}$ is "strongly local" if it satisfies condition L and the following stronger version of WL: 
(SL): For every $p, q \in X$ such that $q \in C(p)$ there exists a connector $\gamma$ joining $p$ and $q$; in particular, the set $C(p)$ is arcwise connected for all $p \in X$.

It is easy to see that if condition $\mathrm{L}$ is satisfied $\mathcal{A}$ must be nondegenerate. Throughout the rest of this paper we will assume that all field theories we discuss are nondegenerate unless noted otherwise.

In physical terms, locality provides for the existence of dynamics, the "finite speed of propagation" of causal signals. Accordingly, condition WL (or SL) guarantees that causal influences propagate from $p$ continuously, and condition L guarantees that signals that communicate with $p$ propagate with "finite speed," and that they connect $p$ to disjoint components of $C(p) \backslash\{p\}$ (which is necessary if dynamics at $p$ is to be determined not only by local evolution equations but also by boundary conditions).

There is a natural notion of "isomorphism" between quantum field theories. Let $X$ be a topological space, and let $\mathcal{A}_{1}$ and $\mathcal{A}_{2}$ be field theories on $X$. Then, $\mathcal{A}_{1}$ and $\mathcal{A}_{2}$ are said to be isomorphic, denoted $\left(\mathcal{A}_{1}, X\right) \cong\left(\mathcal{A}_{2}, X\right)$, if there is a (isometric) $C^{*}$ isomorphism $\Psi: \mathcal{A}_{1} \longrightarrow \mathcal{A}_{2}$ such that for every open subset $U \subset X$,

$$
\mathcal{A}_{2}(U)=\Psi\left[\mathcal{A}_{1}(U)\right] .
$$

As an example, let $\mathcal{A}$ be an ordinary (e.g. Klein-Gordon) field theory on a globally hyperbolic spacetime $(M, g), h: M \longrightarrow M$ be a diffeomorphism of $M$, and define a new theory $h^{*} \mathcal{A}$ on $M$ by (for all open $U \subset M$ )

$$
\left.\left(h^{*} \mathcal{A}\right)(U) \equiv \mathcal{A}^{(K G, m)}\right|_{\left(M, h^{*} g\right)}\left[h^{-1}(U)\right]
$$

[where the field theory that appears on the right-hand side is the standard Klein-Gordon theory corresponding to the spacetime $\left.\left(M, h^{*} g\right)\right]$. It is then easy to see that the theories $\mathcal{A}$ and $h^{*} \mathcal{A}$ thus defined are isomorphic over $M$. Hence our notion of isomorphism is a natural generalization of the usual diffeomorphism invariance in curved-spacetime field theory.

How good a job do the sets $C(p)(p \in M)$ do in providing a generalized "causal structure" on the space $X$ ? A partial answer is given by the following result:

Theorem: Let $X$ be a locally compact topological space, and $\mathcal{A}$ a quantum field theory on $X$. For $U, V \subset X$ open subsets, the subalgebras $\mathcal{A}(U)$ and $\mathcal{A}(V)$ fail to commute if and only if there exists a point $p \in U$ and a point $q \in V$ such that $q \in C(p)$. 
Proof: We first prove the following:

Lemma: Let $X$ be a topological space and $\mathcal{A}$ a quantum field theory on $X$. If $p \in X$ and $U \subset X$ open are such that $\bar{U}$ is compact and $C(p) \cap \bar{U}=\{\}$, then there exists an open neighborhood $V$ of $p$ such that $\mathcal{A}(U)$ and $\mathcal{A}(V)$ commute.

Proof of the Lemma: Since $\bar{U} \cap C(p)=\{\}, \forall q \in \bar{U}$ there exists an open neighborhood $W_{q}$ of $q$ and an open neighborhood $M_{q}$ of $p$ such that $\mathcal{A}\left(W_{q}\right)$ and $\mathcal{A}\left(M_{q}\right)$ commute. Now $\left\{W_{q} \mid q \in \bar{U}\right\}$ is a covering of $\bar{U}$ and $\bar{U}$ is compact; this implies that there exists a finite set of points $q_{1}, \cdots, q_{n} \in \bar{U}$ such that $W_{q_{1}} \cup \cdots \cup W_{q_{n}} \supset \bar{U} \supset U$. But since $\mathcal{A}\left(W_{q_{1}} \cup \cdots \cup W_{q_{n}}\right)=\overline{\left\langle\bigcup_{i=1}^{n} \mathcal{A}\left(W_{q_{i}}\right)>\right.}$, and $\mathcal{A}\left(M_{q_{1}} \cap \cdots \cap M_{q_{n}}\right) \subset \bigcap_{i=1}^{n} \mathcal{A}\left(M_{q_{i}}\right), \quad \mathcal{A}\left(W_{q_{1}} \cup \cdots \cup W_{q_{n}}\right)$ commutes with $\mathcal{A}\left(M_{q_{1}} \cap \cdots \cap M_{q_{n}}\right)$. Thus $\mathcal{A}(U) \subset \mathcal{A}\left(W_{q_{1}} \cup \cdots \cup W_{q_{n}}\right)$ commutes with $\mathcal{A}\left(M_{q_{1}} \cap \cdots \cap M_{q_{n}}\right)$, and $V \equiv M_{q_{1}} \cap \cdots \cap M_{q_{n}}$ is an open neighborhood of $p$ satisfying the desired condition. This completes the proof of the lemma.

Now back to the proof of the theorem. If there exist points $p \in U$ and $q \in V$ such that $q \in C(p)$, then by the definition of $C(p)$ we have that $\mathcal{A}(U)$ and $\mathcal{A}(V)$ must fail to commute. To prove the implication in the converse direction, it clearly suffices to show that if $V \cap \bigcup_{p \in U} C(p)=\{\}$ then $\mathcal{A}(U)$ and $\mathcal{A}(V)$ commute. Let $V \cap \bigcup_{p \in U} C(p)=\{\}$. Since $X$ is locally compact, every $q \in V$ has an open neighborhood $S_{q}$ of compact closure such that $\overline{S_{q}} \subset V$. By the lemma, for each fixed $q \in V$ and given $p \in U$ there exists an open neighborhood $W_{q p}$ of $p$ such that $\mathcal{A}\left(W_{q p}\right)$ and $\mathcal{A}\left(S_{q}\right)$ commute. This implies that $\mathcal{A}\left(S_{q}\right)$ and $\mathcal{A}\left(\bigcup_{p \in U} W_{q p}\right)$ commute, which implies $\mathcal{A}\left(S_{q}\right)$ commutes with $\mathcal{A}(U)$. Since this is true for every $q \in V$ (and the neighborhoods $S_{q}$ cover $V)$, we conclude that $\mathcal{A}(V)$ commutes with $\mathcal{A}(U)$.

Another fundamental causal notion that can be naturally formulated within our general framework is that of "domain of dependence." Namely, an open subset $U \subset X$ is in the domain of dependence of another open set $V \subset X$ if $\mathcal{A}(U)$ is contained in $\mathcal{A}(V)$. Physically, this corresponds to a situation where all fields localized in $U$ can be obtained by evolving fields in $V$ via the local dynamics. To formalize this idea more elegantly, we introduce the following:

Definition: An open subset $U \subset X$ is called a "diamond" if for all open $V \subset X \mathcal{A}(V) \subset \mathcal{A}(U)$ implies $V \subset U$.

Diamonds enjoy a number of properties all of which follow readily from the 
above definition. Namely: (in the following $U, V, W_{\alpha}$ denote open sets in $X)$

(i): The space $X$ is a diamond, and the empty set \{\} is a diamond if and only if $\mathcal{A}$ is nondegenerate.

(ii): If $W_{\alpha}$ are diamonds, then $\operatorname{Int}\left(\bigcap_{\alpha} W_{\alpha}\right)$ is a diamond. Here $\operatorname{Int}(A)$ denotes the topological interior of $A$.

Theorem: $\forall$ open $U \subset X$ there exists a smallest diamond $D(U)$ containing $U$; more precisely, there exists a diamond $D(U)$ such that $D(U) \supset U$ and if $V$ is any other diamond containing $U$ then $V \supset D(U)$.

Proof: $D(U)$ is uniquely given by $\operatorname{Int}\left[\bigcap_{\alpha}\left(D_{\alpha}\right)\right]$, where the intersection is over all diamonds $D_{\alpha}$ containing $U$.

(iii): $U$ is a diamond if and only if $U=D(U)$. Hence $\forall U \quad D(D(U))=D(U)$.

(iv): $D(U)=\bigcup_{\alpha} W_{\alpha}$, where the union is over all open $W_{\alpha}$ such that $\mathcal{A}\left(W_{\alpha}\right) \subset$ $\mathcal{A}(U)$. As a consequence, $\mathcal{A}(D(U))=\mathcal{A}(U) \quad \forall U$.

(v): If $V$ is a diamond and $V \supset U$, then $V \supset D(U)$. From this it follows that $\mathcal{A}(U) \subset \mathcal{A}(V)$ implies $D(U) \subset D(V)$. In particular, $\mathcal{A}(U)=\mathcal{A}(V)$ if and only if $D(U)=D(V)$.

(vi): $D(U \cap V) \subset D(U) \cap D(V)$; equality does not hold in general.

(vii): $D\left(\bigcup_{\alpha} W_{\alpha}\right)=D\left[\bigcup_{\alpha} D\left(W_{\alpha}\right)\right]$.

For an open subset $U \subset X$, the domain of dependence of $U$ is the largest open set in which all local fields are dynamically determined by the fields localized in $U$. It is clear from the above properties that the diamond $D(U)$ is precisely the domain of dependence.

We started this discussion by pointing out that in quantum field theory the structure consisting of $\mathcal{A}$ and the subalgebras $\{\mathcal{A}(U)\}$ is the fundamental observable associated with spacetime topology, the sought-after analogue in quantum theory of the observable $\mathcal{F}$ of classical physics. But we have not yet explained how the topological space $(X, \tau)$ can be recovered from a knowledge of $[\mathcal{A},\{\mathcal{A}(U)\}]$. Indeed, this task now appears quite nontrivial: it seems impossible to distinguish, solely by observing the subalgebras $\mathcal{A}(U)$, the open 
subsets $U \subset X$ from their associated diamonds $D(U)$, since, as we have seen above, $\mathcal{A}(U)=\mathcal{A}(D(U))$ for every open set. The true observable, then, is the collection $\{\mathcal{A}(D)\}$, where $D$ ranges over all diamonds in $X$. How can we reconstruct $(X, \tau)$ using only the structure of its diamonds?

We will answer this question shortly. First, however, we will study the essential structure in the observables $\{\mathcal{A}(U)\}$ and $\{\mathcal{A}(D)\}$ which contains the information relevant to spacetime topology; namely, the partial order on the sets $\{\mathcal{A}(U)\}$ and $\{\mathcal{A}(D)\}$, given by open-set inclusion, $U \subset U^{\prime}$, in the first case, and diamond inclusion, $D \subset D^{\prime}$, in the second [by (v) above, the partial order on $\{\mathcal{A}(D)\}$ in this second case coincides with that given by subalgebra inclusion]. More precisely, the structure of $\{\mathcal{A}(U)\}$ is that of a " $C^{*}$ frame," and the structure of $\{\mathcal{A}(D)\}$ is that of a " $C^{*}$ lattice." This leads us naturally into our final, ultimate generalization of quantum field theory: The structure of the observables $\{\mathcal{A}(U)\}$ [or of $\{\mathcal{A}(D)\}]$ makes no explicit reference to the background topological space $(X, \tau)$. In quantum field theory, the fundamental information about spacetime structure is contained in the partial ordering of the set $\{\mathcal{A}(U)\}$, and, therefore, it is natural to consider the category of such ordered-subalgebras as the proper domain to which spacetime structure ultimately belongs in quantum theory.

Let us, then, proceed to a general discussion of this new category.

\section{Quantum lattices and quantum frames}

We assume throughout this section that the reader is familiar with the basic notions of lattice theory, including frames and their connection with topological spaces. For a systematic treatment of these subjects, see [8] and [9]; alternatively, the Appendix below presents a quick review of the relevant concepts. The background contained in the Appendix is sufficient for following our discussion in this section.

A $C^{*}$ lattice is a complete lattice $L$ which, as a set, consists of closed subalgebras of an abstract $C^{*}$ algebra $\mathcal{A}$ (with identity), and where the partial order on $L$, given by the usual subalgebra inclusion, satisfies the following properties: (i) the least element $\mathbf{0}$ is $\mathbf{C}$ and the largest element $\mathbf{1}$ is $\mathcal{A}$, and

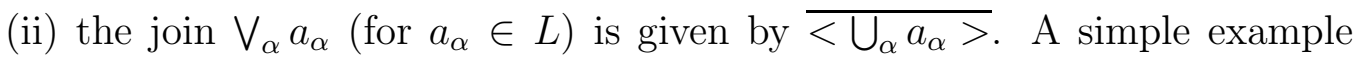
of a $C^{*}$ lattice is the complete lattice $M$ of all closed subalgebras of a $C^{*}$ algebra $\mathcal{A}$ (with identity). In $M$, the meet operation is simply $\bigwedge_{\alpha} a_{\alpha}=\bigcap_{\alpha} a_{\alpha}$. Note that for a more general $C^{*}$ lattice $L$ based on $\mathcal{A}$ the meet in $L$ would not, in general, have this simple form; in other words, $L$ is not necessarily 
a sublattice of $M$ (see Appendix), even though the partial order on $L$ is induced from that on $M$. More nontrivial examples of $C^{*}$ lattices are those given by $\{\mathcal{A}(D)\}$, where $\mathcal{A}$ is a quantum field theory on a topological space $X$ and $D$ ranges over all diamonds in $X$ (see Sect. 3 ).

A $C^{*}$ frame $(F, \mathcal{A})$ consists of a frame $F$, an abstract $C^{*}$ algebra $\mathcal{A}$ (with identity), and a map $\mathcal{A}$ that associates to each $b \in F$ a closed subalgebra $\mathcal{A}(b) \subset \mathcal{A}$ such that the conditions

$$
\mathcal{A}(\mathbf{0})=\mathrm{C}, \quad \mathcal{A}(\mathbf{1})=\mathcal{A},
$$

and

$$
\mathcal{A}\left(\bigvee_{\alpha} b_{\alpha}\right)=\overline{<\bigcup_{\alpha} \mathcal{A}\left(b_{\alpha}\right)>}, \quad b_{\alpha} \in F
$$

are satisfied. Typical examples of quantum frames are, of course, those in which $F$ is the open-set frame $\Omega(X)$ of a topological space $X$, and the map $\mathcal{A}$ associates to each open set $b \in F$ the local field algebra $\mathcal{A}(b)$ of a quantum field theory $\mathcal{A}$ on $X$. Recall (see Appendix) that given a frame $F$, we have a canonical construction which associates to $F$ a topological space $\operatorname{pt}(F)$. As long as the space $X$ is "reflexive" (see Appendix), it can be reconstructed from its open-set frame $\Omega(X)$ as pt $[\Omega(X)]$. For example, all Hausdorff $X$ are reflexive. Therefore, provided spacetime is at least Hausdorff, its topology can be completely recovered from the observable $[\mathcal{A},\{\mathcal{A}(U)\}]$ simply by using the frame structure of the $C^{*}$ frame $\{\mathcal{A}(U)\}$. In other words, for a reflexive $X$, a quantum field theory $\mathcal{A}$ on $X$ contains precisely the same information as the open-set frame $\Omega(X)$, endowed with the $C^{*}$-frame structure given by the theory $\mathcal{A}$. This is the reconstruction we promised at the end of the last section; it is the quantum analogue of the reconstruction of a Tychonoff $X$ from the classical observable $\mathcal{F}$ (see Sect. 2). On the other hand, the natural category to which the notion of "spacetime structure" belongs now becomes the category of frames and frame maps, manifestly larger than the category of topological spaces and continuous functions. Indeed, there is no reason to assume a priori that $F$ for a $C^{*}$ frame $(F, \mathcal{A})$ is the open-set frame of any topological space; in general it is not, as $F$ coincides with $\Omega[\operatorname{pt}(F)]$ only for a restricted class of frames (called "spatial" frames; see Appendix). We will discuss some of the implications of this generalization in the next paper of this series (see the concluding section below). In the remainder of this paper, we will briefly explore some elementary properties of quantum lattices and frames; but first, we will pursue the answer to the second question posed at the end of the last section: namely the reconstruction of $X$ from the observable $\{\mathcal{A}(D)\}$ (where $D$ ranges over the diamonds in $X$ ). 
We have seen that $\{\mathcal{A}(D)\}$ has the structure of a $C^{*}$ lattice, and that there is a canonical construction which associates to each frame $F$ a topological space pt $(F)$. The reconstruction we desire would be described (for sufficiently regular spaces $X$ ) once we describe how to construct a $C^{*}$ frame $(F, \mathcal{A})$ canonically associated to every $C^{*}$ lattice $L$ (based on $\mathcal{A}$ ). We will now present such a construction:

Let $L$ be any complete lattice. We first describe how to construct a frame $F(L)$ canonically associated to $L$. The main idea here is to see the lattice $L$ as a "tiling" of the frame $F(L)$. Think of the following concrete example as a model: Let $F$ be the open-set frame of the Euclidean space $\mathbf{R}^{2}$, and let $L$ be the lattice of all open rectangles, i.e. open sets in $\mathbf{R}^{2}$ of the form $I \times J$, where $I$ and $J$ are open intervals in $\mathbf{R}$ (partial order on $L$ is the usual inclusion, and the meet is $\bigwedge_{\alpha} U_{\alpha}=\operatorname{Int}\left(\bigcap_{\alpha} U_{\alpha}\right)$; the same as the meet operation in the open-set frame $F$ ). In this example, there is a clear intuitive sense in which $L$ "tiles" $F$, and it seems obvious that $F$ should be constructible entirely in terms of $L$. Our construction is an abstract scheme in which this idea is made precise.

So given an arbitrary complete lattice $L$ with join $\vee$ and meet $\wedge$, let $2^{L}$ denote the set of all subsets of $L$, and introduce an equivalence relation $\sim$ on $2^{L}$ by

$$
\text { for } K, K^{\prime} \subset L, \quad K \sim K^{\prime} \Longleftrightarrow \bigvee_{k \in K} x \wedge k=\bigvee_{k^{\prime} \in K^{\prime}} x \wedge k^{\prime} \forall x \in L .
$$

There is a natural partial order on the quotient set $2^{L} / \sim$, namely

$$
[K] \leq\left[K^{\prime}\right] \Longleftrightarrow \bigvee_{k \in K} x \wedge k \leq \bigvee_{k^{\prime} \in K^{\prime}} x \wedge k^{\prime} \quad \forall x \in L,
$$

where $[K]$ denotes the equivalence class in $2^{L} / \sim$ of the subset $K \in 2^{L}$, and the order $\leq$ on the right hand side is that of the original lattice $L$. The definition (7) is clearly independent of which representatives $K, K^{\prime}$ are chosen for the classes $[K]$ and $\left[K^{\prime}\right]$ [see Eq. (6)]. Now put $F(L) \equiv 2^{L} / \sim$. It is easy to verify that the set $F(L)$ under the partial order $(7)$ is a complete lattice, and that the join $\vee^{F}$ and meet $\wedge^{F}$ of $F(L)$ are simply $[K] \vee^{F}\left[K^{\prime}\right]=\left[K \cup K^{\prime}\right]$ and $[K] \wedge^{F}\left[K^{\prime}\right]=\left[K \wedge K^{\prime}\right]$, where $K \wedge K^{\prime}$ denotes the subset $K \wedge K^{\prime} \equiv$ $\left\{k \wedge k^{\prime} \mid k \in K, k^{\prime} \in K^{\prime}\right\}$. In fact we have, more generally,

$$
\bigvee_{\alpha}^{F}\left[K_{\alpha}\right]=\left[\bigcup_{\alpha} K_{\alpha}\right], \quad \bigwedge_{\alpha}^{F}\left[K_{\alpha}\right]=\left[\bigwedge_{\alpha} K_{\alpha}\right]
$$

where $\left[K_{\alpha}\right]$ are an arbitrary collection of elements of $F(L)$. [It is a pleasant exercise to verify that the definitions (8) do not depend on which representatives $K_{\alpha}$ are chosen for the classes $\left[K_{\alpha}\right] \in F(L)$.] It is also obvious that 
the least element $\mathbf{0}$ of $F(L)$ is $[\{\}]$ and the largest element $\mathbf{1}$ is $[L]$. We now claim that $F(L)$ with this complete lattice structure is, in fact, a frame, i.e. satisfies the join-infinite distributive identity [Appendix, Eq. (12)]. To see this, we simply observe that for $[M],\left[K_{\alpha}\right] \in F(L)$,

$$
\begin{aligned}
{[M] \wedge^{F} \bigvee_{\alpha}^{F}\left[K_{\alpha}\right] } & =\left[M \wedge \bigcup_{\alpha} K_{\alpha}\right]=\left[\bigcup_{\alpha} M \wedge K_{\alpha}\right] \\
& =\bigvee_{\alpha}^{F}\left[M \wedge K_{\alpha}\right]=\bigvee_{\alpha}^{F}[M] \wedge^{F}\left[K_{\alpha}\right] .
\end{aligned}
$$

There exists a natural imbedding $i: L \longrightarrow F(L)$ given by $i: k \in L \mapsto[\{k\}] \in$ $F(L)$. It is not difficult to show that $i$ is one-to-one and order preserving in both directions, i.e. $k \leq k^{\prime} \Longleftrightarrow i(k) \leq i\left(k^{\prime}\right)$. Thus $L$ is imbedded in $F(L)$ in such a way that the order on $L$ coincides with that induced from $F(L)$, and the meet $\wedge$ of $L$ coincides with the meet $\wedge^{F}$ of $F(L)$. Moreover, $F(L)$ is generated by $L$ under this imbedding since, clearly, $\forall[K] \in F(L)$ we have $[K]=\left[\bigcup_{k \in K} k\right]=\bigvee^{F}{ }_{k \in K}[\{k\}]$. Hence the lattice $L$ is imbedded in $F(L)$ as a "tiling," just like the example we described in the previous paragraph. Note, also, that if $L$ itself is a frame, then $F(L)$ simply coincides with $L$ [see Eqs. (6) - (7)]. This completes our construction of the frame $F(L)$ associated to an arbitrary lattice $L$.

Now let $L$ be a $C^{*}$ lattice based on a $C^{*}$ algebra $\mathcal{A}$. Construct the frame $F(L)$ associated to $L$ as in the above paragraph. Each element $b$ of $F(L)$ is an equivalence class $[A]$ of subsets of $L$. Moreover, by Eq. (6), for any two representatives $A, A^{\prime}$ of $[A]$ we have $\bigvee_{a \in A} a=\bigvee_{a^{\prime} \in A^{\prime}} a^{\prime}$ [just take $x=1$ in Eq. (6)]. For a $C^{*}$ lattice $L$, where the join is simply the closure of the subalgebra generated by its arguments, this implies that $\overline{\left\langle\bigcup_{a \in A} a\right\rangle}$ is a well defined $C^{*}$ algebra for each $b=[A]$ in $F(L)$. The assignment of this algebra to each element $b$ of the frame $F(L)$ gives $F(L)$ the structure of a $C^{*}$ frame [see Eqs. $(7)$ and (8)]. This is the canonical $C^{*}$ frame $[F(L), \mathcal{A}]$ associated to the $C^{*}$ lattice $L$.

It is also possible to go back from a $C^{*}$ frame $(F, \mathcal{A})$ to a $C^{*}$ lattice $L(F)$ of "diamonds" of $F$, where, in the context of a $C^{*}$ lattice $(F, \mathcal{A})$, diamonds are defined analogously to Sect. 3 as elements $d \in F$ such that $\forall b \in F$ $\mathcal{A}(b) \subset \mathcal{A}(d)$ implies $b \leq d$.

Finally, we come to the formal definition of "quantum frames" and "quantum lattices." A quantum frame is essentially a $C^{*}$ frame $(F, \mathcal{A})$, with the additional technical conditions that (i) $F$ is generated by its atoms, (ii) $\forall b \in F, b<\mathbf{1}$, there exists a completely prime filter disjoint from $j^{-}(b) \equiv$ $\{d \in F \mid d \leq b\}$ (see Appendix for the definitions of these terms), and (iii) 
$\mathcal{A}(b)$ is a central $C^{*}$ algebra $\forall b \in F$. A quantum lattice is a $C^{*}$ lattice $L$ whose associated $C^{*}$ frame $(F, \mathcal{A})$ is a quantum frame. These extra restrictions prove useful in discussing certain physical properties of quantum frames and lattices, as we will see when we study examples of these objects in our next paper of the series.

For a foretaste of the kind of physics one can study in this framework, let us try to define a notion of locality for quantum frames. Let $(F, \mathcal{A})$ be a quantum frame, and for any $b \in F$ define the "causal complement" $S(b)$ of $b$ as the set

$$
S(b) \equiv\{d \in F \mid[\mathcal{A}(d), \mathcal{A}(b)]=0\} .
$$

Define the "causal trace" of $b$ as the subset

$$
J(b) \equiv\left\{d \in F \mid j^{-}(d) \cap S(b)=\{\mathbf{0}\}\right\} .
$$

Recall that for a subset $A \subset F, \vee A$ denotes the element $\bigvee_{a \in A} a$. Let for each $b \in F \tilde{b}$ denote the "exterior" of $b$ defined by $\tilde{b} \equiv \bigvee\{d \in F \mid d \wedge b=\mathbf{0}\}$. Now we can define a quantum frame $(F, \mathcal{A})$ to be local if it has the following two properties:

(i) For every atom $b \in F$ the element $\bigvee J(b)$ is an atom.

(ii) Every completely prime filter $\mathcal{P}$ in $F$ admits a basis $\mathcal{B}$ and an element $f \in \mathcal{P}$ such that for each $e \in \mathcal{B}$ the element $f \wedge \tilde{e} \wedge \vee J(e)$ is composite.

It is not hard to see that this definition is designed to be as close as possible within the frame category to the general notion of locality introduced in Sect. 3 for topological spaces and quantum fields. Note, also, that we can define a local quantum lattice simply as a quantum lattice whose associated quantum frame is local.

As a last remark, we note that just as in the case of the general framework of Sect.3, so here also we have a natural generalization of the notion of "diffeomorphism invariance" in terms of the notion of isomorphism between quantum lattices and frames. An isomorphism between two quantum lattices (or general $C^{*}$ lattices) $L_{1}$ and $L_{2}$ is defined simply as a $C^{*}$-algebra isomorphism $\Psi: \mathcal{A}_{1} \longrightarrow \mathcal{A}_{2}$ which carries the subalgebras that constitute $L_{1}$ onto those that constitute $L_{2}$. Similarly, an isomorphism between two quantum frames (or more general $C^{*}$ frames) $\left(F_{1}, \mathcal{A}_{1}\right)$ and $\left(F_{2}, \mathcal{A}_{2}\right)$ is a pair $(f, \Psi)$, where $f: F_{1} \longrightarrow F_{2}$ is a frame isomorphism, $\Psi: \mathcal{A}_{1} \longrightarrow \mathcal{A}_{2}$ is a $C^{*}$ algebra 
isomorphism, and the two are compatible in the sense that

$$
\Psi\left[\mathcal{A}_{1}(b)\right]=\mathcal{A}_{2}[f(b)]
$$

for all $b \in F_{1}$.

\section{Conclusion}

We have finally obtained a quite promising, if not entirely satisfactory, answer to the original question posed in the Introduction; namely, we now have a rather precise understanding of the physical origin of spacetime topology, or, more generally, of spacetime structure. The most promising aspect of our approach so far is the fact that it naturally suggests a candidate for the fundamental, "discrete" structure of spacetime at arbitrarily small length scales, namely the structure of a quantum lattice or frame. There are a number of advantages to this suggestion for the discrete structure of spacetime: For one, our formalism does not treat spacetime as a set of "points" equipped with some (topological, causal, ...) preferred structure. Points of a frame $F$ do not have fundamental physical reality, even though they may be constructed abstractly as "completely prime filters" in the lattice structure of $F$. This is in agreement with the intuitive expectation that in full quantum gravity "topology fluctuations" would prevent one from defining spacetime points in a sensible way. Also, in our approach the notion of "diffeomorphism invariance" finds a very natural and simple reformulation in terms of isomorphisms of quantum frames. And, finally, quantum frames have a quantized structure at the outset; no extra "quantization" step is necessary to study their implications for the small-scale structure of spacetime.

Of course, much remains to be done before we can determine whether our approach can ever contribute to the larger goal of gaining new insights into quantum gravity. Here we focused our attention mainly on developing the mathematical foundations for our viewpoint; hence our discussion has been rather general and abstract. In the next paper ([10]) of this series we will tackle some of the more "physical" questions raised by our discussion above. Among the subjects of this forthcoming manuscript are a discussion of quantum lattices generated by the usual quantum field theory models in curved spacetime, the analysis of some simple toy-models for "discrete" quantum frames and lattices, and a discussion on the role and possible quantization of the "metric" in our approach.

\section{Appendix: Some basic facts about lattices and frames}


What we will give here is mainly an explanation of the terminology used and the statements (without proofs) of the results referred to in the discussion of Sect. 4. We refer the reader to the sources [8] and [9] for proofs and for more detailed information.

A lattice is a set $L$ with a partial order $\leq$ such that for every pair of elements $a, b \in L$ the (unique) least upper bound, l.u.b.(a,b), and the greatest lower bound, g.l.b $(a, b)$, both exist. These elements are denoted by $a \vee b$, and $a \wedge b$, respectively, and both $\vee$ (called the "join") and $\wedge$ (called the "meet") are commutative, associative binary operations on $L$. A complete lattice is a lattice in which the meet and join of arbitrary (not just finite) collections of elements exist. A frame is a complete lattice $F$ in which the join-infinite distributive identity

$$
a \wedge \bigvee_{\alpha} b_{\alpha}=\bigvee_{\alpha} a \wedge b_{\alpha}, \quad \forall a, b_{\alpha} \in F
$$

holds. If $A \subset L$ is a subset of a complete lattice $L$, we denote by $\bigvee A$ and $\wedge A$ the elements $\bigvee_{a \in A} a$ and $\bigwedge_{a \in A} a$ of $L$. In any complete lattice $L$, there is defined a least element $\mathbf{0} \equiv \wedge L$, and a largest element $\mathbf{1} \equiv \bigvee L$. An example of a complete lattice is the lattice of all subsets of a set $A$ partially ordered by inclusion. In this case, $\vee$ and $\wedge$ coincide with the set union $\cup$ and set intersection $\cap$, respectively. This lattice is always a frame. For an example of a complete lattice which is not a frame, consider the lattice of all open rectangles in $\mathbf{R}^{2}$ discussed in Sect. 4 . Other typical examples of frames are given by the open-set lattices, denoted $\Omega(X)$, of topological spaces $(X, \tau)$. In $\Omega(X)$, the join is the usual set union, but the meet is given by $\bigwedge_{\alpha} U_{\alpha}=\operatorname{Int}\left(\bigcap_{\alpha} U_{\alpha}\right)$, which generally coincides with set intersection only for finite collections $\left\{U_{\alpha}\right\}$ of open sets.

Let $L_{1}, L_{2}$ be lattices, and $f: L_{1} \longrightarrow L_{2}$ be a map. Then, denoting by $a, a_{\alpha}$ and $b$ arbitrary elements of $L_{1}$, we can introduce the following notions:

(i) $f$ is called order preserving if $a \leq b \Longrightarrow f(a) \leq f(b)$.

(ii) $f$ is order preserving in both directions if $a \leq b \Longleftrightarrow f(a) \leq f(b)$.

(iii) $f$ is a lattice homomorphism if $f(a \vee b)=f(a) \vee f(b)$ and $f(a \wedge b)=$ $f(a) \wedge f(b)$. If $L_{1}$ and $L_{2}$ are complete, then $f$ is a complete lattice homomorphism if $f\left(\bigvee_{\alpha} a_{\alpha}\right)=\bigvee_{\alpha} f\left(a_{\alpha}\right)$ and $f\left(\bigwedge_{\alpha} a_{\alpha}\right)=\bigwedge_{\alpha} f\left(a_{\alpha}\right)$.

(iv) $f$ is a lattice isomorphism if it is one-to-one, onto, and a lattice homomorphism; or, equivalently, if it is onto and order preserving in both 
directions.

(v) If $L_{1}$ and $L_{2}$ are frames, then $f$ is a frame map if it is a lattice homomorphism that preserves arbitrary joins and maps $\mathbf{0} \mapsto \mathbf{0}$ and $\mathbf{1} \mapsto \mathbf{1}$. $f$ is a frame isomorphism if it is both a frame map and a lattice isomorphism.

Notice that frame maps generalize inverses of continuous functions; i.e., if $X, Y$ are topological spaces and $f: X \longrightarrow Y$ is a continuous map, then $f^{-1}: \Omega(Y) \longrightarrow \Omega(X)$ is a frame map. Hence the category of frames and frame maps is a generalization of the category of topological spaces and continuos functions.

In any lattice $L$, for $a \in L j^{-}(a)$ denotes the subset $j^{-}(a)=\{b \in$ $L \mid b \leq a\}$, and, more generally, for a subset $A \subset L j^{-}(A)$ is defined as $j^{-}(A) \equiv\{b \in L \mid \exists a \in A$ such that $b \leq a\} \cdot j^{+}(a)$ and $j^{+}(A)$ are defined similarly. An element $a \in L$ is called an atom if $a$ cannot be written in the form $b \vee c$ with $b \wedge c=\mathbf{0}$; if $a$ can be written in this form it is called composite.

Let $\{0,1\}$ denote the unique frame with two elements. If $F$ is any frame, we define

$$
\operatorname{pt}(F) \equiv\{p: F \longrightarrow\{0,1\} \mid p \text { is a frame map }\} .
$$

The elements of $\operatorname{pt}(F)$ are called the points of $F$. Now consider all subsets of the set $\operatorname{pt}(F)$ which have the form $\mathcal{O}_{b} \equiv\{p \in \operatorname{pt}(F) \mid p(b)=1\}$ for some $b \in$ $F$. It is not hard to show that the collection of subsets $\left\{\mathcal{O}_{b} \subset \operatorname{pt}(F) \mid b \in F\right\}$ defines a topology on $\operatorname{pt}(F)$. By $\operatorname{pt}(F)$ we will always denote this canonical topological space associated to the frame $F$. It follows that $\operatorname{pt}(F)$ is always $T_{0}$. A frame $F$ is called spatial if $F$ is frame-isomorphic to $\Omega[\operatorname{pt}(F)]$. A topological space $X$ is called reflexive if $X$ is homeomorphic to $\operatorname{pt}[\Omega(X)]$. It can be shown that all Hausdorff spaces are reflexive.

Let $L$ be an arbitrary lattice. A subset $S \subset L$ is called a sublattice if $S$ is closed under the operations $\wedge$ and $\vee$ of $L$. A subset $P \subset L$ is called a filter if $a, b \in P$ implies $a \wedge b \in P$ and $a \in P, b \geq a$ implies $b \in P$. Equivalently, a filter $P$ is a sublattice for which $a \in P$ implies $a \vee b \in P$ for all $b \in L$. For any element $a \in L$, the set $j^{+}(a)$ is a filter. Let $P \subset L$ be a filter. A basis for $P$ is a subset $\mathcal{B} \subset L$ such that $a \in P \Longleftrightarrow a \geq b$ for some $b \in \mathcal{B}$; in other words, $P=j^{+}(\mathcal{B})$. A filter $P \subset L$ is called prime if for all $a, b \in L \quad a \vee b \in P$ implies either $a \in P$ or $b \in P$. For example, in the lattice of all subsets of a set $A$, filters of the form $j^{+}(\{a\})$ are prime for all $a \in A$. If 
$L$ is a complete lattice and $P \subset A$ is a filter, $P$ is called completely prime if for any arbitrary collection $\left\{a_{\alpha}\right\} \subset L \bigvee_{\alpha} a_{\alpha} \in P$ implies that at least one of the $a_{\alpha}$ is contained in $P$. The filters $j^{+}(\{a\})$ in the example we have just given are completely prime. It can be shown that, for a frame $F$, a subset $\mathcal{P} \subset F$ is a completely prime filter if and only if there exists a frame map $f: F \longrightarrow\{0,1\}$ such that $\mathcal{P}=f^{-1}(1)$. In other words, the set $\operatorname{pt}(F)$ of "points" of a frame $F$ is in one-to-one correspondence with the set $\{\mathcal{P}\}$ of all completely prime filters in $F$. 


\section{REFERENCES}

1. R. Penrose, in Quantum Theory and Beyond, Ted Bastin ed. (Cambridge University Press, Cambridge 1971).

2. L. Bombelli, L. Lee, D. Meyer and R. Sorkin, Phys. Rev. Letters 59, 521 (1987); ibid. 60, 656 (1987).

3. C. J. Isham, An Introduction to General Topology and Quantum Topology, lectures presented at the Advanced Summer Institute on Physics, Geometry and Topology, Banff, August 1989.

4. S. Willard, General Topology (Addison-Wesley, Reading, Mass. 1970).

5. B. S. Kay and R. M. Wald, Phys. Reports 207, 49 (1991).

6. R. M. Wald, in: Quantum Mechanics in Curved Spacetime, J. Audretsch and V. de Sabbata eds. (Plenum, New York, 1992).

7. U. Yurtsever, Algebraic approach to quantum field theory in non-globallyhyperbolic spacetimes, UCSB Physics preprint UCSBTH-92-43, October 1992.

8. G. Graetzer, General Lattice Theory (Birkhauser, Basel 1978).

9. P. T. Johnstone, Stone Spaces (Cambridge University Press, Cambridge 1982).

10. U. Yurtsever, Quantum lattices and quantum frames, UCSB Physics Preprint (in preparation). 